\title{
Statins reduce the burden of ambient particulate matter and inflammatory cells within the lung tissues of smokers with and without COPD
}

\author{
To the Editor:
}

Previous studies from our laboratories have shown retention of particulate matter (PM) in lung tissues of subjects with chronic obstructive pulmonary disease (COPD), with increased retention in more severe COPD, potentially contributing to the persistent lung inflammation and remodelling of lung tissue in COPD [1]. Epidemiological studies have shown that 3-hydroxy-3-methylglutaryl coenzyme A reductase inhibitors (statins) reduce morbidity and mortality in subjects with COPD [2], possibly via their well-known pleiotropic anti-inflammatory properties $[3,4]$. In an animal model we have shown that statins reduce lung inflammation induced by ambient PM exposure in part by reducing the retention of $\mathrm{PM}$ in lung tissues by promoting their removal via the lymphatic system to regional lymph nodes $[5,6]$. We postulated that statins reduce the burden of PM retention in the lung tissues of subjects with COPD, and thereby reduce downstream lung inflammatory responses.

We quantified the burden of PM in lung tissues (from the lung tissue registry at the Centre for Heart Lung Innovation, Vancouver, BC, Canada) of smokers with and without COPD, who used statins for $\geqslant 3$ months for comorbid hyperlipidaemia, prior to their lobectomy or pneumonectomy for a small peripheral lung tumour. Lung tissues were processed as described in detail previously [7]. We used standard morphometric methodology previously described [1] to quantify the burden of PM in different lung compartments (total PM burden in all lung tissues and PM burden in alveolar walls, macrophages, blood vessel walls and lymphoid tissues). To determine the effect of statins on the downstream inflammatory response in the lung we quantified neutrophils and macrophages, two pivotal innate immune effector cells in COPD pathogenesis, in the same lung tissues. Using immunohistochemistry, neutrophils were labelled with neutrophil elastase primary antibody (Dako Laboratories, Glostrup, Denmark) and all lung macrophages were labelled using a double immunostaining technique using anti-CD68 (Dako). Anti-CD206 (GeneTex, Irvine, CA, USA) was used to identify a subpopulation of M2 macrophages. Morphometric methods (point counting) were used to quantify neutrophils and macrophages present in lung tissues $[1,7]$.

Samples from 21 subjects who used statins and 43 subjects not using statins prior to surgery were selected to determine the burden of PM in lung tissues. Demographic parameters between the groups were not significantly different with regards to sex, smoking history (pack-years) or severity of COPD (forced expiratory volume in $1 \mathrm{~s}(\mathrm{FEV} 1)$ and $\mathrm{FEV} 1 /$ forced vital capacity (FVC) ratio). The statin group was slightly older that the nonstatin group (mean \pm SD age $70.3 \pm 5.1$ years versus $63.1 \pm 10.1$ years, $\mathrm{p}<0.01$ ). Atorvastatin, rosuvastatin and simvastatin were the three statins most commonly used by the study subjects. The total PM burden was higher in lung tissues of COPD subjects versus subjects with normal spirometry. The total PM burden in lung tissues in the statin users was lower (figure $1 \mathrm{a} ; \mathrm{p}=0.0058$ ) and this decrease was predominantly due to a decrease in $\mathrm{PM}$ in alveolar walls (figure $1 \mathrm{~b} ; \mathrm{p}=0.0304$ ) and alveolar macrophages (figure $1 \mathrm{c} ; \mathrm{p}=0.0341$ ). The most striking difference was in the vasculature, where statins reduced the PM volume fraction in blood vessel walls (figure 1d; $\mathrm{p}<0.0001$ ), and this reduction was observed in smokers with and without COPD ( $\mathrm{p}=0.0032$ and $\mathrm{p}=0.0037$, respectively). The volume fractions of lymphoid tissues in both groups were not significantly different. Lung inflammatory cells were quantified in a subset of 16 statin users and compared to 14 subjects not using statins,

@ERSpublications

Statins promote the processing and removal of inhaled particles from lung tissues, thereby reducing lung inflammation http://ow.ly/Zx68305bv3k

Cite this article as: Hiraiwa K, Miller S, Ngan DA, et al. Statins reduce the burden of ambient particulate matter and inflammatory cells within the lung tissues of smokers with and without COPD. Eur Respir J 2017; 49: 1601689 [https://doi.org/10.1183/13993003.01689-2016]. 

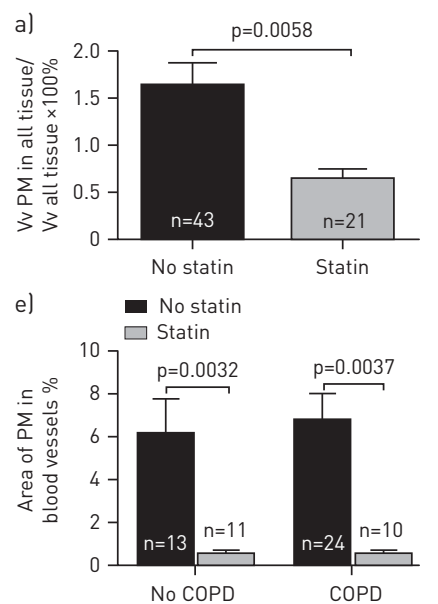

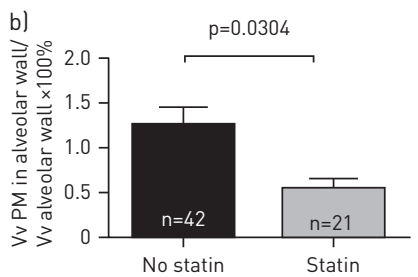

f)

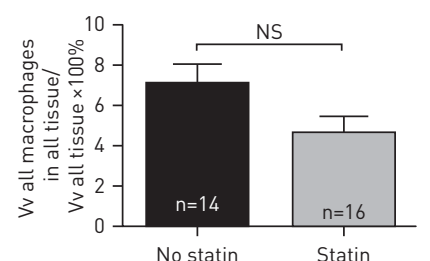

c)

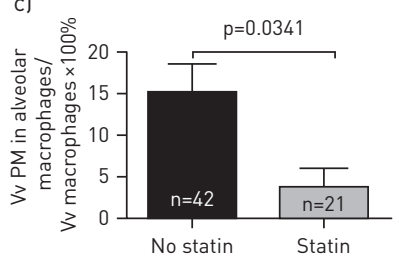

g)

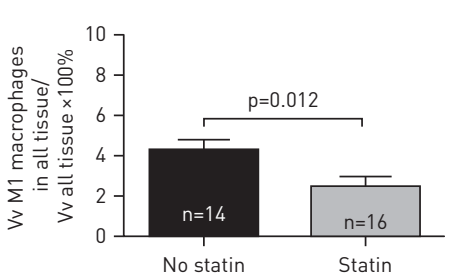

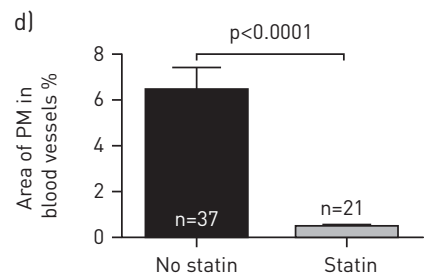

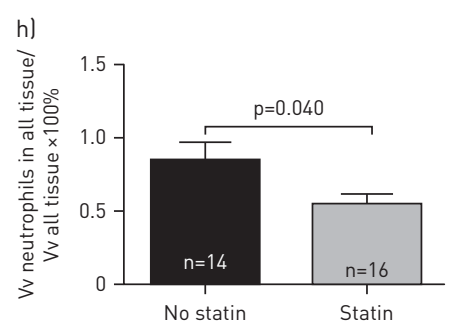

FIGURE 1 Statins reduce particulate matter (PM) burden in lung tissues. a) Statins significantly lower the total PM burden when compared to the nonstatin treatment. The lung tissue compartments that showed the most significant reduction in PM volume fractions (Vv) were b) alveolar walls, c) alveolar macrophages and d) blood vessel walls. e) In blood vessel walls the burden of PM was equally reduced in non-chronic obstructive pulmonary disease (COPD) versus COPD subjects. The $\mathrm{V} v$ of fl all macrophages in lung tissues was not different between groups, but gl M1 skewed macrophages and h) neutrophils were lower in statin users. Data are presented as mean \pm SE.

matched for severity of COPD. In statin users, the volume fraction of neutrophils in the lung tissues was significantly reduced compared to subjects not using statins $(\mathrm{p}=0.04)$ and this reduction was predominantly observed in the airway wall tissues $(\mathrm{p}=0.012)$. In statin users the volume fraction of CD206-negative macrophages (M1 skewed) in the lung tissues was reduced compared to subjects not using statins ( $\mathrm{p}=0.012)$. There was a significantly negative correlation between the burden of PM in lung tissues and the FEV1/FVC ratio, as well as the magnitude of smoking exposure (pack-years), and statins attenuate these correlations.

This study shows that statin treatment reduces the burden of PM in the lung tissues of smokers with and without COPD. We have previously quantified the PM burden in lung tissues of subjects with COPD [1], showing that the PM burden increases with COPD severity, suggesting that diseased lungs have a reduced ability to clear PM from the lung and postulating that retained PM in lung tissues could potentiate inflammatory responses in the COPD lung $[1,8]$. In human studies, statins are known to be associated with reduced morbidity and mortality in variety of chronic inflammatory lung conditions, including COPD [2, 9], effects thought to be due to their well-known pleiotropic or anti-inflammatory properties impacting lung inflammatory responses $[3,4]$. We have shown in animal studies that statins reduce the burden of PM retained in lung tissues, in particular in lung macrophages, and increase PM burden in regional lymphoid tissues. This suggests that one potential mechanism for the reduction of PM burden in lung tissues by statins might be enhanced removal of PM-loaded macrophages to regional lymph nodes [5]. In addition, in these animal studies we showed that statins reduced lung inflammatory response, predominantly neutrophil recruitment into airspaces and lung tissues, induced by the exposure to ambient PM. Here we showed decreased neutrophil inflammation in lung tissues in statin-using smokers with and without COPD, which supported our observations in animal studies. In addition, we showed skewing of macrophages towards an M1 phenotype in the lung tissues of statin users. Both neutrophils and macrophages are immune cells strongly implicated in the pathogenesis and progression of COPD [7, 10-12].

The retention of PM in lung tissues, in macrophages in particular, impairs their phagocytic ability to clear foreign material, including PM, bacterial pathogens and apoptotic cells from the lung in COPD [12, 13], which could contribute to the persistence of lung tissue inflammation that characterises COPD [1]. Statins enhance macrophage efferocytosis [3] and thereby promote clearance of apoptotic debris and cells (such as neutrophils) within lung tissues; they also accelerate the processing and clearance of PM particles from lung tissues and thereby reduce lung inflammation. Augmenting macrophage functional properties in COPD would provide a novel therapeutic pathway that could reduce lung inflammation and progression of COPD.

Changes in pulmonary vessels are an important component in the pathogenesis of COPD progression [14, 15], and our study showed a dramatic reduction of the volume fraction of PM in the pulmonary blood vessel walls, both in smokers with normal lung function as well as subjects with COPD. This effect of statins on blood vessel PM burden suggests that this class of anti-inflammatory intervention could possibly also attenuate vascular remodelling in COPD through reducing deposits of PM around lung vessels. Together, our study suggests that statins promote the processing and removal of PM from the lung tissues, and we speculate that this reduced burden of PM in lung tissues results in an attenuated inflammatory response within the lung 
(supported by our findings of fewer neutrophils and pro-inflammatory macrophages in lung tissues). Clearly, further mechanistic studies are needed, but our results support the notion that statin treatment could be a potential therapeutic strategy to slow the progression of COPD.

Kunihiko Hiraiwa, Sally Miller, David A. Ngan, Dragoș M. Vasilescu, Tillie-Louise Hackett, Daisuke Kinose, Jui Chih Cheng and Stephan F. van Eeden

University of British Columbia, Centre for Heart and Lung Innovation, St Paul's Hospital, Vancouver, BC, Canada.

Correspondence: Stephan F. van Eeden, University of British Columbia, Centre for Heart and Lung Innovation, St. Paul's Hospital, 1081 Burrard Street, Vancouver, BC, V6Z 1Y6, Canada. E-mail: Stephan.vanEeden@hli.ubc.ca

Received: June 32016 | Accepted after revision: Oct 102016

Support statement: This research was undertaken with financial support from the British Columbia Lung Association. S. van Eeden is supported by a scholarship from the Canadian Institutes of Health Research/GlaxoSmithKline (CIHR/GSK professorship in COPD). Funding information for this article has been deposited with the Open Funder Registry.

Conflict of interest: Disclosures can be found alongside this article at erj.ersjournals.com

Acknowledgements: We would like to thank all the laboratory technicians and summer students who contributed to this work.

\section{References}

1 Ling SH, McDonough JE, Gosselink JV, et al. Patterns of retention of particulate matter in lung tissues of patients with COPD: potential role in disease progression. Chest 2011; 140: 1540-1549.

2 Cao $\mathrm{C}, \mathrm{Wu} \mathrm{Y}, \mathrm{Xu} \mathrm{Z}$, et al. The effect of statins on chronic obstructive pulmonary disease exacerbation and mortality: a systematic review and meta-analysis of observational research. Sci Rep 2015; 5: 16461.

3 Morimoto K, Janssen WJ, Fessler MB, et al. Lovastatin enhances clearance of apoptotic cells (efferocytosis) with implications for chronic obstructive pulmonary disease. J Immunol 2006; 176: 7657-7665.

4 Jasińska M, Owczarek J, Orszulak-Michalak D. Statins: a new insight into their mechanisms of action and consequent pleiotropic effects. Pharmacol Rep 2007; 59: 483-499.

5 Miyata R, Bai N, Vincent R, et al. Statins reduce ambient particulate matter-induced lung inflammation by promoting the clearance of particulate matter $<10 \mu \mathrm{m}$ from lung tissues. Chest 2013; 143: 452-460.

6 Miyata R, Bai N, Vincent R, et al. Novel properties of statins: suppression of the systemic and bone marrow responses induced by exposure to ambient particulate matter $\left(\mathrm{PM}_{10}\right)$ air pollution. Am J Physiol Lung Cell Mol Physiol 2012; 303: L492-L499.

7 Hogg JC, Chu F, Utokaparch S, et al. The nature of small-airway obstruction in chronic obstructive pulmonary disease. N Engl J Med 2004; 350: 2645-2653.

8 van Eeden SF, Tan WC, Suwa T, et al. Cytokines involved in the systemic inflammatory response induced by exposure to particulate matter air pollutants $\left(\mathrm{PM}_{10}\right)$. Am J Respir Crit Care Med 2001; 164: 826-830.

9 Thomson NC. Clinical studies of statins in asthma and COPD. Curr Mol Pharmacol 2016 [In press DOI: 10.2174/ 1874467209666160112125911]

10 Russell DW, Wells JM, Blalock JE. Disease phenotyping in chronic obstructive pulmonary disease: the neutrophilic endotype. Curr Opin Pulm Med 2016; 22: 91-99.

11 Nurwidya F, Damayanti T, Yunus F. The role of innate and adaptive immune cells in the immunopathogenesis of chronic obstructive pulmonary disease. Tuberc Respir Dis 2016; 79: 5-13.

12 Shaykhiev R, Krause A, Salit J, et al. Smoking-dependent reprogramming of alveolar macrophage polarization: implication for pathogenesis of chronic obstructive pulmonary disease. J Immunol 2009; 183: 2867-2883.

13 Lundborg M, Dahlén SE, Johard U, et al. Aggregates of ultrafine particles impair phagocytosis of microorganisms by human alveolar macrophages. Environ Res 2006; 100: 197-204.

14 Pinkerton KE, Green FH, Saiki C, et al. Distribution of particulate matter and tissue remodeling in the human lung. Environ Health Perspect 2000; 108: 1063-1069.

15 Peinado VI, Pizarro S, Barberà JA. Pulmonary vascular involvement in COPD. Chest 2008; 134: 808-814. 